\title{
Salicylate enhances the response of prostate cancer to radiotherapy.
}

Lindsay A. Broadfield ${ }^{4 *}$, Katarina Marcinko ${ }^{4 *}$, Evangelia Tsakiridis ${ }^{4,5}$, Panayiotis G. Zacharidis $^{4,5}$, Linda Villani ${ }^{4}$, James Lally ${ }^{4}$, Gabe Menjolian ${ }^{2}$, Danitra Maharaj ${ }^{2}$, Tammy Mathurin ${ }^{2}$, Marcia Smoke ${ }^{2}$, Thomas Farrell ${ }^{3}$, Paola Muti ${ }^{5}$, Gregory R. Steinberg ${ }^{4}$ and Theodoros Tsakiridis $1,5,6 @$

${ }^{1}$ Divisions of Radiation Oncology, ${ }^{2}$ Radiotherapy and ${ }^{3}$ Physics, Juravinski Cancer Center, ${ }^{4}$ Division of Endocrinology and Metabolism, Department of Medicine and Departments of ${ }^{5}$ Oncology and ${ }^{6}$ Pathology and Molecular Medicine, McMaster University

@ Address Correspondence to:

Theos Tsakiridis, M.D., Ph.D.

Radiation Oncology, Juravinski Cancer Center

Dept. of Oncology, McMaster University

699 Concession Street,

Hamilton, ON, Canada, L8V 5C2

This is the author manuscript accepted for publication and has undergone full peer review but has not been through the copyediting, typesetting, pagination and proofreading process, which may lead to differences between this version and the Version of Record. Please cite this article as doi: 10.1002/pros. 23755

This article is protected by copyright. All rights reserved 
* These authors contributed equally to this work

\begin{abstract}
Background: Radiotherapy (RT) is a key therapeutic modality for prostate cancer (PrCa), but RT resistance necessitates dose-escalation, often causing bladder and rectal toxicity. Aspirin, a prodrug of salicylate (SAL), has been associated with improved RT response in clinical PrCa cases, but the potential mechanism mediating this effect is unknown. SAL activates the metabolic stress sensor AMP-activated protein kinase (AMPK), which inhibits de novo lipogenesis, and protein synthesis via inhibition of Acetyl-CoA Carboxylase (ACC), and the mammalian Target of Rapamycin (mTOR), respectively. RT also activates AMPK through a mechanism distinctly different from SAL. Therefore, combining these two therapies may have synergistic effects on suppressing PrCa. Here, we examined the potential of SAL to enhance the response of human PrCa cells and tumors to RT.
\end{abstract}

Methods: Androgen-insensitive (PC3) and -sensitive (LNCaP) PrCa cells were subjected to proliferation and clonogenic survival assays after treatment with clinically relevant doses of SAL and RT. Balb/c nude mice with PC3 xenografts were fed standard chow diet or chow diet supplemented with $2.5 \mathrm{~g} / \mathrm{kg}$ salsalate (SAL pro-drug dimer) one week prior to a single dose of 0 or 10Gy RT. Immunoblotting analysis of signaling events in the DNA repair and AMPK-mTOR pathways and lipogenesis were assessed in cells treated with SAL and RT.

Results: SAL inhibited proliferation and clonogenic survival in PrCa cells and enhanced the inhibition mediated by RT. Salsalate, added to diet, enhanced the anti-tumor effects of RT in PC3 tumor xenografts. RT activated genotoxic stress markers and the activity of mTOR pathway and AMPK and mediated inhibitory phosphorylation of ACC. Interestingly, SAL enhanced the effects of RT on AMPK and ACC but blocked markers of mTOR activation.

This article is protected by copyright. All rights reserved 
Conclusions: Our results show that SAL can enhance RT responses in PrCa. Salsalate is a promising agent to investigate this concept in prospective clinical trials of $\mathrm{PrCa}$ in combination with RT.

\section{Introduction}

Radiotherapy (RT) is key therapeutic modality for all stages of prostate cancer (PrCa), but these tumors are radio-resistant and require escalating RT doses to improve local control ${ }^{1,2}$. This imposes a therapeutic challenge since dose-escalation leads to increased bladder and rectal toxicities $^{1,2}$. There is need to improve the therapeutic ratio of RT in PrCa. Ideal therapies would be well-tolerated agents that could improve the local control achieved with RT and may allow dose de-escalation.

A significant body of retrospective data suggests that aspirin (acetyl-salicylate), the most widely used anti-inflammatory agent, is associated with reduced risk for $\mathrm{PrCa}^{3-10}$, PrCa-related death ${ }^{11,12}$ and improved outcomes in patients treated with $\mathrm{RT}^{13,14}$. Analysis of about six thousand men participating in the Cancer of the Prostate Strategic Urologic Research Endeavor (CaPSURE), showed that aspirin was associated with reduced PrCa mortality in men treated with RT or prostatectomy ${ }^{15}$. In smaller retrospective studies, aspirin, and other anti-coagulants, were associated with improved biochemical control (assessed via Prostate Specific Antigen; PSA) and reduced risk for distant metastasis at 4 years after curative $\mathrm{RT}^{13}$. Jacobs et al. $(2014)^{16}$ observed that aspirin and anti-coagulants were associated with improved freedom from biochemical failure in African Americans with high risk (Gleason 9-10) PrCa treated with curative RT. Similarly, Osbon et al. (2016) ${ }^{17}$ found that aspirin was associated with reduction in biochemical recurrence and distant metastasis-free survival at 7 years, in patients treated with curative RT. In localized early-stage PrCa treated with RT alone, lack of aspirin use was associated with shorter interval to This article is protected by copyright. All rights reserved 
biochemical failure $(<18 \text { months })^{18}$. Finally, aspirin was associated with improved health-related quality of life in patients treated with RT but, unfortunately, it was also associated with increased risk for rectal bleeding ${ }^{19}$.

Upon ingestion of aspirin (acetyl-salicylate) the acetyl-group is cleaved by carboxyesterases and it is this acetyl group which inhibits prostaglandin synthesis and is vital for its anticoagulant activity (for review see ${ }^{20}$ ). However, this does not occur with the ingestion of salsalate $^{21}$, a dimer of salicylate (SAL), which has been used extensively for the treatment of rheumatoid arthritis and is currently in clinical testing for type 2 diabetes and cardiovascular disease $^{21}$. Given the absence of anti-platelet effects, salsalate can be delivered at much higher concentrations (4-5g/day) leading to SAL serum concentrations of approximately $1 \mathrm{mM}$, compared to approximately $10 \mu \mathrm{M}$ in patients on 'baby' $(75 \mathrm{mg}$ ) aspirin therapy (for review see $\left.{ }^{20}\right)$. Importantly, at serum concentrations achievable with therapeutic salsalate doses $(>500 \mu \mathrm{M})$, SAL directly binds to and increases the activity of the metabolic stress sensor AMP-activated protein kinase (AMPK) ${ }^{22}$, an enzyme that is associated with decreased cancer growth in multiple cancer types and is a potential target for cancer therapy ${ }^{23}$. AMPK is a heterotrimeric enzyme comprised of $\alpha$-catalytic and $\beta$ - and $\gamma$-regulatory subunits ${ }^{24,25}$. Salicylate binds to the AMPK $\beta 1$ subunit leading to allosteric activation of the catalytic $\alpha$-subunit ${ }^{22,26}$. The tumor suppressor Liver Kinase B1 (LKB1), also activates AMPK through phosphorylation of the $\alpha$-subunit at Thr17225.

Activated AMPK may suppress cancer cell growth through multiple pathways. This includes inhibiting mTOR complex 1 (mTORC1) through phosphorylation of Tuberous Sclerosis 2 (TSC2) and Raptor (Ser792), which suppresses the ribosomal activity and translation initiation via inhibition of p70 S6 kinase $\left(\mathrm{p} 70^{\mathrm{S} 6 \mathrm{~K}}\right)$ and the eukaryotic initiation factor $4 \mathrm{E}$ binding protein 1 
$(4 \mathrm{EBP} 1)^{27}$, respectively. In addition, AMPK inhibits de novo lipogenesis (DNL), which is vital for membrane biosynthesis and proliferation. This occurs through phosphorylation-mediated blockade of the rate-limiting enzymes of fatty acid and cholesterol synthesis pathways AcetylCoA carboxylase 1 and $2(\mathrm{ACC} 1 / 2)$ and $\beta$-Hydroxy- $\beta$-Methylglutaryl-CoA Reductase (HMGCR), respectively ${ }^{25,28}$.

Further, during metabolic stress, AMPK suppresses metabolic gene expression and the cell cycle through phosphorylation (Ser15) and activation of $p 53^{29}$. Interestingly, AMPK also responds to genotoxic stress downstream of DNA Damage Response (DDR) signals initiated by Ataxia Telengiectasia Mutated (ATM), to induce p53 and mediate the ionizing radiation-induced G2-M checkpoint and suppression of survival ${ }^{30}$.

Previously, we found that SAL suppresses clonogenic survival of PrCa cells in vitro, effects associated with activation of AMPK and suppression of mTOR and DNL ${ }^{26}$. With the clinical observations of aspirin benefiting PrCa patients treated with RT and the ability of SAL to activate AMPK, inhibit DNL, and suppress PrCa growth led us to conduct our present study. The aims of the this work were to: 1) investigate the effects of salicylate in combination with RT in PrCa cells ( $\mathrm{LNCaP}$ and PC3) in vitro and 2) examine whether salicylate delivered orally in the form of salsalate could enhance the effects of RT in vivo in PC3 PrCa xenografts.

\section{Materials and Methods}

Cell lines and treatments. PrCa cells ( $\mathrm{PC} 3, \mathrm{LNCaP})$ were from the American Type Culture Collection (ATCC). The normal human prostate epithelial PNT1A cells were a kind gift from Dr. Gurmit Singh, McMaster University. Cells were grown in Roswell Park Memorial Institute 
(RPMI) medium with $1 \%$ antibiotic-anti-mycotic (Gibco) and $10 \%$ FBS (Gibco), at $37^{\circ} \mathrm{C}$. Cells were treated with sodium-salicylate (SAL) (Sigma) and/or RT as indicated.

Proliferation assays. Cells were seeded in 96-well plates and allowed to adhere overnight. SAL treatments were applied after $24 \mathrm{~h}$ and RT or sham treatments 24-hours later. Cells were allowed to grow for 5 days, washed and stained with $0.5 \%$ crystal violet stain (Sigma). Plates were dried overnight, cells were solubilized in $0.05 \mathrm{M} \mathrm{NaH} \mathrm{PO}_{4}$ in $50 \%$ ethanol and optical density was analyzed.

Clonogenic assays. Cells were seeded into 12-well plates, allowed to adhere overnight. SAL pretreatment occurred 24-hours prior to RT, and cells grew for 7 (PC3) or 14 (LNCaP and PNT1A) days. Cells were then fixed and stained with crystal violet. Colonies $>50$ cells were counted, and colony counts were normalized to untreated controls.

Lipogenesis assay. Cells were pretreated without or with SAL for 24-hours followed by RT (0 or 2Gy). 48-hours later cells were incubated with radio-labeled ${ }^{3} \mathrm{H}$-sodium acetate $(10 \mu \mathrm{Ci} / \mathrm{ml}$, PerkinElmer) and unlabeled sodium acetate $(0.5 \mathrm{mM})$ for 4-hours, were washed with cold PBS, scraped and subjected to chloroform-methanol lipid extraction. Radio-labelled lipids were counted in a scintillator, as described ${ }^{28}$.

Xenograft experiments. 5-week old male BALB/c-Nude mice (Charles-River: Mississauga, ON) were housed in a pathogen-free facility with ad libitum access to radiated chow and water, under a 12-hour light/dark cycle. PC3 cells $\left(1 \times 10^{6}\right)$ in warm PBS were grafted subcutaneously into the flank. When tumor volumes approached $100 \mathrm{~mm}^{3}$, animals were randomly divided into four groups: (1) chow, (2) salsalate (2.5g/kg in chow diet), (3) RT-chow, (4) RT-salsalate, six animals per group. One week after starting diet treatments, mice were subjected to isoflurane anesthesia, transferred to HEPA filter Plexiglas tubes and were treated with a single fraction of 0 or 10 Gy of This article is protected by copyright. All rights reserved 
RT using clinical Linear Accelerators and an established conformal technique and dosimetry ${ }^{31}$. Non-RT treated mice and RT-treated mice were sacrificed after 7 or 8 weeks, respectively. Actual tumor volumes were measured at euthanasia. Harvested tumors were Formalin Fixed Paraffin Embedded (FFPE), cross-sectioned and stained with H+E. All animal procedures were approved by McMaster University Animal Research Ethics Board.

Immunoblotting. Cells grown in 6-well plates were treated with SAL and/or RT as indicated. One-hour or 48-hours after treatments, cells were washed, lysed in protease and phosphatase inhibitor containing buffer. Protein levels were evaluated and lysates were subjected to SDSPAGE and immunoblotting, as described ${ }^{26,31}$, using specific primary antibodies and horseradish peroxidase (HRP)-conjugated secondary antibodies. All antibodies were from Cell Signaling (New England Biolabs, Mississauga, ON). Blots were imaged using Clarity Wester ECL (BioRad) and quantified using ImageJ Software (NIH). All blots are normalized to $\beta$-actin loading controls for each gel.

Statistical analysis. Results are expressed as a mean with standard error of the mean (SEM). $p<0.05$ was considered significant. Prism-6 statistical software was used to run two-way ANOVA, and Fisher LSD multiple comparisons test. Quadratic linear curve fitting was used to describe dose-response relationships. Inhibitory (50\%) concentration ( $\left.\mathrm{IC}_{50}\right)$ values were calculated using this data transformed to $\log (\mathrm{x})$ and the variable slope nonlinear fit calculation in Prism. Combination Index (CI) analysis was pursued with the Chou-Talalay method, using the CompuSyn software, as described ${ }^{31-33}$. 


\section{Results}

Salicylate enhances RT-mediated inhibition of $\operatorname{PrCa}$ proliferation and clonogenic survival.

We began this work with evaluation of effects of SAL on proliferation rates. Increasing doses of SAL suppressed proliferation in control and irradiated PC3 cells. Importantly, SAL inhibited proliferation at clinically achievable high $\mu \mathrm{M} \operatorname{doses}^{26}$ and RT fractions of 2-4 Gy (Fig.1A-B). IC 50 values for SAL, RT, and SAL with 2 Gy RT were calculated to be $972.5 \mu \mathrm{M}$

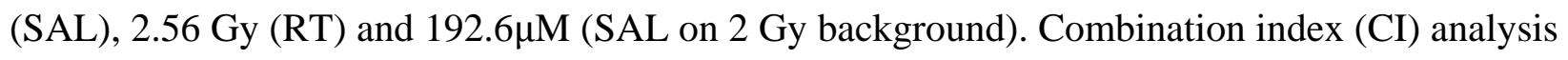

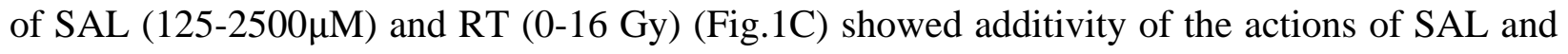
RT at low doses of the two agents (see Table S1 in supplemental results, for details). SAL induced a synergistic suppression of proliferation with $2-8$ Gy of RT, with $1 \mathrm{mM} \mathrm{SAL}+4$ Gy RT being an optimal combination (see CI values, Table s1). Synergy between the two agents is lost at very high doses of either SAL or RT.

Next, we aimed to analyze the effects of SAL on clonogenic (oncogenic) capacity of androgen-insensitive PC3 and androgen-sensitive LNCaP cells, without or with combined treatment with RT. However, control clonogenic experiments were performed first with the human normal prostate epithelial line PNT1A. In PNT1A cells low $(50 \mu \mathrm{M})$ or high $(500$ and $1000 \mu \mathrm{M})$ SAL doses did not affect significantly clonogenic survival (Fig.s1). A trend for suppression of survival (mean $15 \%$ inhibition) was detected at $1000 \mu \mathrm{M}$ but this did not reach significance. This lack of efficacy of SAL on PNT1A clonogenic survival, even at doses that block PrCa cell proliferation, indicated the expected very low cytotoxic capacity of this drug against non-cancer tissue. For that, no further experiments were performed with those cells. However, in both PC3 and LNCap cells, SAL inhibited clonogenic survival alone and in 
combination with RT (2 Gy). Effects of SAL alone became statistically significant at $100 \mu \mathrm{M}$. Survival was inhibited at lower drug doses compared to proliferation (Fig.1A-E). PC3 cells were less sensitive to SAL alone and in combination with $\mathrm{RT}$ compared to $\mathrm{LNCaP}$ cells, with $\mathrm{IC}_{50}$ values of $506.6 \mu \mathrm{M}(0 \mathrm{~Gy})$ and $315.8 \mu \mathrm{M}(2 \mathrm{~Gy})$ in PC3 cells and $306 \mu \mathrm{M}(0 \mathrm{~Gy})$ and $73.1 \mu \mathrm{M}(2$ Gy) in LNCaP cells. RT (2 Gy) suppressed survival in both PC3 and LNCaP cells (46\% and 41\% of untreated controls) and SAL enhanced the response to 2 Gy RT in both cell lines. Finally, we examined low dose SAL in combination with increasing RT doses. PC3 cells were more resistant to RT compared to $\mathrm{LNCaP}$ but both cell lines showed consistent trends for improved RT

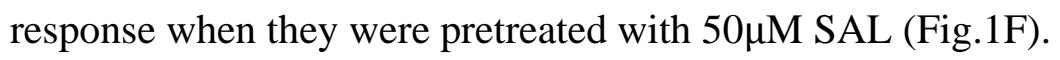

Salsalate enhances the anti-tumor effects of RT in vivo.

Effects of the salicylate dimer, salsalate, and RT in vivo were examined in PC3 xenografts. Grafted animals were provided chow diet without or with $2.5 \mathrm{~g} / \mathrm{kg}$ salsalate (achieving circulating salicylate levels of $900 \mu \mathrm{M}^{34}$ ) and/or treated with RT (10 Gy) (Fig.2A). In non-irradiated tumors, salsalate did not alter tumor growth kinetics (Fig.2B). RT alone reduced tumor volume compared to controls (average $311.1 \mathrm{~mm}^{3}$ vs $1478.8 \mathrm{~mm}^{3}$, respectively) (Fig.2B,C). Importantly, salsalate enhanced the growth inhibitory effects of RT $\left(97.5 \mathrm{~mm}^{3} \mathrm{vs} 311.1 \mathrm{~mm}^{3}\right.$, respectively) (Fig.2C). RT reduced significantly PC3 tumor volumes. Recovery took place 5 weeks later, while in salsalate-fed animals, tumor volume remained low until endpoint (Fig.2C). Consistent with in-vivo evaluations, ex-vivo direct measurement of tumor volumes, at time of euthanasia, also showed suppression of tumor growth with addition of salsalate diet to RT 
treatment (Fig.2D). Representative tumor cross sections of FFPE tumors, stained with H+E, are shown (Fig.2E).

Salicylate and RT affects regulation of AMPK-mTOR signaling events.

AMPK, a negative regulator of mTOR signaling, is activated by RT downstream of $\mathrm{ATM}^{24,30}$. In PC3 cells, we observed an acute (1-hour) activation of the ATM-AMPK axis by RT alone, which was sustained for 48-hours, as measured by elevated ACC phosphorylation (Fig.3A-C). RT also led to sustained activation of the mTOR pathway, detected as $\mathrm{p} 70^{\mathrm{S} 6 \mathrm{~K}}$ activation (S6 phosphorylation) and phosphorylation of 4EBP1, suggesting enhanced protein synthesis for cell repair (Fig.3D-E). SAL treatment elevated levels of inhibitory phosphorylation of ACC in non-irradiated PC3 cells (Fig.3F,G). Importantly, SAL treatment enhanced AMPK activation and, although it did not further increase ACC phosphorylation, it almost completely blocked $\mathrm{p} 70^{\mathrm{S} 6 \mathrm{~K}}$ and 4EBP1 phosphorylation (Fig.3H,I). These effects were sustained chronically (48h) with enhanced AMPK $\alpha$ (T172) phosphorylation, inhibitory phosphorylation of ACC and Raptor and inhibition of $\mathrm{p} 70^{\mathrm{S} 6 \mathrm{~K}}$ in control and irradiated cells (Fig.3J-L).

Salicylate inhibits de novo lipogenesis (DNL) in PC3 cells.

To analyze the impact of ACC1/2 (Ser79/212) phosphorylation, DNL was examined. SAL $(500 \mu \mathrm{M}$ and $1 \mathrm{mM})$ caused dose-dependent inhibition of DNL in both non-irradiated cells and irradiated cells, albeit to a lesser extent (Fig.3M). However, we could not demonstrate increased suppression of DNL by the drug in irradiated cells. 


\section{Discussion}

Despite the extensive body of retrospective clinical evidence suggesting an association of aspirin with improved PrCa outcomes, very few preclinical studies examined the effects of aspirin in PrCa models and even fewer focused specifically on SAL. High dose SAL (0.5$20 \mathrm{mM}$ ) was shown to suppress growth in PrCa cell models ${ }^{35,36}$ and block endothelial to mesenchymal transition (EMT) in PC3 cells ${ }^{37}$. In cervical cancer SAL was shown to enhance the anti-proliferative and pro-apoptotic effects of $\mathrm{RT}^{38}$ but, to date, SAL has not been investigated in combination with RT in pre-clinical models of PrCa.

\section{SAL enhances RT response in PrCa cells}

Here, we show that SAL inhibits proliferation and survival of PC3 and LNCaP PrCa cells at clinically achievable doses $(125-1000 \mu \mathrm{M})$ and enhances the cytotoxicity of RT (Fig.1). Importantly, SAL mediates this anti-tumor action without significant activity on normal human prostate epithelial cells (Fig. s1), indicating a potential of this drug to provide an improved therapeutic ratio. In PrCa cells, we demonstrate additivity and synergy in the anti-proliferative effects of SAL and RT indicating a strong potential for combined SAL+RT treatment to improve the clinical outcomes of RT (see CI values Fig.1C, Table S1). The inhibitory effect of SAL on PrCa cell proliferation diminished at higher doses of RT (>8 Gy) (Fig.1B). Similarly, the antiproliferative action of RT was limited at higher SAL doses (Fig.1A), indicating that the two agents may engage some overlapping mechanisms. SAL (1mM) showed similar antiproliferative efficacy with that of 2 Gy RT indicating a potential for RT dose reduction with combined therapy. The enhanced synergy of SAL with 4 Gy radiotherapy $(\mathrm{CI}=0.51$, Fig.1C, 
Table s1) suggests that the optimal drug use may be the combination of SAL with hypofractionated RT, which is a new standard of care for $\mathrm{PrCa}^{39}$. Importantly, the efficacy of SAL to suppress clonogenic survival in irradiated cells with IC50 values of $73.1 \mu \mathrm{M}$ (LNCaP) to $315.8 \mu \mathrm{M}$ (PC3) (Figs.1D-E), illustrates the potential of significantly lower, well-tolerated, drug levels to improve the response of $\mathrm{PrCa}$ to RT. This is supported by our observation that drug concentrations as low as $50 \mu \mathrm{M}$ show trends for PrCa sensitization to increasing fractions of $\mathrm{RT}$ (Fig.1F).

Salsalate enhaces prostate tumor response to $R T$.

Since clinical trials showed that serum SAL levels of approximately $1 \mathrm{mM}$ can be achieved in human circulation with well-tolerated oral doses of salsalate ${ }^{40-42}$, we fed immunocompromised animals grafted with PC3 tumors with salsalate-supplemented chow diet $(2.5 \mathrm{~g} / \mathrm{Kg})$, known to achieve in mice serum SAL levels of $900 \mu \mathrm{M}^{34}$.

Despite the observed inhibition of proliferation and clonogenic survival of PrCa cells invitro by SAL treatment alone, we could not observe suppression of PC3 xenograft growth in animals treated with salsalate diet alone. Currently, we do not have an explanation for this observation. It is possible that the tumor micro-environment provides mechanisms of resistance to salicylate action, which are inhibited or blocked by RT treatment allowing uncovering of the salicylate anti-tumor activity in irradiated tumors only. These may involve tumor vascular supply and levels of hypoxia, which can be investigated in future studies.

Salsalate diet enhanced tumor response to RT (Fig. 2C). Irradiated tumors sustained growth inhibition but showed resistance to RT and recovery of growth 5 weeks later. This 
resistance of PrCa tumors to RT was largerly abolished with salsalate diet. Currently, the exact mechanism of cell death in tumors receiving combined treatment is unclear. We hypothesized that salsalate diet may mediate additional tumor suppression in irradiated tumors through induction of necrosis. Detailed analysis of 2-dimentional images, obtained with microscopy of $\mathrm{H}+\mathrm{E}$ stained sections from all extracted tumors at end-point, was performed. Interestingly, although some necrosis was detected in tumors from untreated and salsalate treated animals, this was reduced in irradiated tumors and those treated with salsalate + RT (Fig. s2). The proportion of high and normal cellular density increased slightly with RT and SAL treatment. In addition to major reduction in the overall size and cross-sectional area, tumors from RT and salsalate + RT treated animals showed proportional reduction in areas of fibrosis and necrosis and were populated with dense pyknotic nuclei (Fig. s2). Such tumor morphology would not be unexpected at end-point after RT and salsalate treatment when phagocytosis is expected to have cleared necrotic cells. Pycknotic cellular appearance may indicate that residual tumors cells may have undergone senescence or destined for apoptosis.

Overall, the above results show that salsalate is a promising agent that may be able to abolish RT resistance in human PrCa and it should be investigated clinically in prospective trials in combination with RT.

\section{Mechanism of action of SAL.}

Earlier studies with aspirin observed that suppression of $\mathrm{PrCa}$ proliferation was associated with inhibition of the survival pathways of Stat3 and Erk1/2 35 . We found no significant modulation of Erk1/2 phosphorylation by SAL in our studies (Fig. s3A). Earlier work from our group provided convincing evidence that SAL, i) binds directly to Ser108 of the AMPK 
$\beta 1$ subunit leading to allosteric activation of the AMPK enzymatic complex ${ }^{22}$ and ii) suppresses PrCa survival through a mechanism that requires AMPK $\beta 1$ and involves suppression of $\mathrm{DNL}^{26}$. In this study we demonstrate once again that SAL, i) activates AMPK, ii) mediates inhibitory phosphorylation of ACC and suppression of DNL and ii) blocks the mTORC1 pathway, indicated by, inhibitory phosphorylation of Raptor (S792) and blockade of the activating Thr389 phosphorylation on $\mathrm{p} 70^{\mathrm{S} 6 \mathrm{~K}}$ (Fig.3F,J). These effects are observed early (within $1 \mathrm{~h}$ ) and are sustained in the long term $(48 \mathrm{~h})$, indicating that SAL mediates an effective suppression of DNL and mTORC1-mediated growth. The specific phosphorylation sites of Ser79/212 on ACC and Ser792 on Raptor are established targets of $\mathrm{AMPK}^{28}{ }^{43}$. Therefore, these results are consistent with our earlier observations ${ }^{26}$ and point to the central role of AMPK in the regulation of metabolic events by SAL in PrCa cells.

\section{Effects in irradiated cells}

Similar to earlier work ${ }^{24,44}$, this study found that RT activates ATM and AMPK but also enhances acutely the activity of the mTORC1 pathway (Fig.3). However, SAL induced a robust suppression of RT-mediated stimulation of the mTORC1 pathway, inhibiting phosphorylation of p70 ${ }^{\mathrm{S} 6 \mathrm{~K}}$, S6, and 4EBP1 in irradiated cells (Fig.3F,H,I). These effects of SAL were also sustained in the long term. At $48 \mathrm{~h}$ after RT, SAL pre-treated cells maintained enhanced inhibitory phosphorylation of S792 on Raptor and reduced T389 phosphorylation of p70 ${ }^{\mathrm{S} 6 \mathrm{~K}}$ (Fig.3J). Now, there is solid evidence that mTORC1 regulates Cap-dependent translation, ribosomal biogenesis and expression of cell cycle, DNL and glycolysis genes, leading to a tight control of protein synthesis, cellular growth and survival ${ }^{45,46}$. Many of these key functions of mTORC1 are 
mediated through $\mathrm{p} 70^{\mathrm{S} 6 \mathrm{k}}$ and $4 \mathrm{EBP} 1^{45,46}$. Our observations that SAL mediates an effective blockade on $\mathrm{p} 70^{\mathrm{S} 6 \mathrm{k}}$ and 4EBP1 regulation in non-irradiated and irradiated $\mathrm{PrCa}$ cells and that it eliminates the activation of mTORC1 effectors by RT (Fig.3F-L), indicates the strong potential of this drug to effectively down-regulate ribosomal function and protein synthesis ${ }^{47}$ in $\mathrm{PrCa}$ treated with RT. This effect of SAL could indeed lead to improved clinical outcomes of RT in this disease.

SAL also induced sustained inhibitory phosphorylation of ACC1/2 (Ser79/212) and suppression of DNL in irradiated cells (Fig.3J-M). We have shown that the SAL-mediated suppression of DNL is responsible for the suppression of PrCa cell survival, since fatty acid add back experiments produced a significant reversal of the effects of the drug ${ }^{26}$. However, RT had no measurable effect on lipogenic rates in cells pre-treated with SAL. These results suggest that the additional inhibition of proliferation and clonogenic survival mediated by RT in SAL-treated cells is mediated mainly by DNL-independent mechanisms.

Since p53 is a key regulator of cell cycle progression, apoptosis and survival, we examined its regulation by SAL in untreated and irradiated LNCaP cells expressing wild-type p53. In these cells, RT induced acute (1h) phosphorylation of ATM and p53 (Ser15) (Fig.s3B). However, SAL was not able to increase this activity beyond the effect of RT, indicating that modulation of p53 is likely not a key pathway for the SAL-induced enhancement of RT toxicity.

Taken together, the findings of this study point that the mTORC1 pathway, an established driver of growth, survival and resistance to cytotoxic therapies, is suppressed by SAL in PrCa cells to mediate the enhanced response of these cells to RT. 


\section{Conclusions}

The current study shows that SAL, a metabolite of salsalate, decreases proliferation and clonogenic survival of PrCa and enhances the anti-tumor activity of $\mathrm{RT}$ in vitro and in vivo. This is accomplished with concentrations of SAL that can be achieved safely in human serum with oral administration of the pro-drug salsalate. Our findings suggest that in irradiated cells SAL triggers a mechanism of action that involves activation of AMPK and suppression of ACC and the mTORC1 pathway leading to an observed enhancement of RT cytotoxicity. This is illustrated in the model of Figure 3N. These observations support strongly investigation of salsalate in combination with RT in randomized clinical trials in localized PrCa, where there is a need for improvement of the therapeutic ratio of RT.

Acknowledgments: This study was supported by a grant from the Canadian Association of Radiation Oncologists, ACURA program.

Conflict of Interest: The authors declare no conflicts of interest. 
References:

1. Zietman AL, Bae K, Slater JD, et al. Randomized trial comparing conventional-dose with high-dose conformal radiation therapy in early-stage adenocarcinoma of the prostate: long-term results from proton radiation oncology group/american college of radiology 95-09. J Clin Oncol. 2010;28(7):1106-1111.

2. Zietman AL, DeSilvio ML, Slater JD, et al. Comparison of conventional-dose vs high-dose conformal radiation therapy in clinically localized adenocarcinoma of the prostate: a randomized controlled trial. Jama. 2005;294(10):1233-1239.

3. Dasgupta K, Di Cesar D, Ghosn J, Rajan R, Mahmud S, Rahme E. Association between nonsteroidal anti-inflammatory drugs and prostate cancer occurrence. Cancer J. 2006;12(2):130-135.

4. Garcia Rodriguez LA, Gonzalez-Perez A. Inverse association between nonsteroidal antiinflammatory drugs and prostate cancer. Cancer Epidemiol Biomarkers Prev. 2004;13(4):649-653.

5. Huang TB, Yan Y, Guo ZF, et al. Aspirin use and the risk of prostate cancer: a metaanalysis of 24 epidemiologic studies. Int Urol Nephrol. 2014;46(9):1715-1728.

6. Jacobs EJ, Rodriguez C, Mondul AM, et al. A large cohort study of aspirin and other nonsteroidal anti-inflammatory drugs and prostate cancer incidence. J Natl Cancer Inst. 2005;97(13):975-980.

7. Perron L, Bairati I, Moore L, Meyer F. Dosage, duration and timing of nonsteroidal antiinflammatory drug use and risk of prostate cancer. Int J Cancer. 2003;106(3):409415.

8. Salinas CA, Kwon EM, FitzGerald LM, et al. Use of aspirin and other nonsteroidal antiinflammatory medications in relation to prostate cancer risk. Am J Epidemiol. 2010;172(5):578-590.

9. Shebl FM, Sakoda LC, Black A, et al. Aspirin but not ibuprofen use is associated with reduced risk of prostate cancer: a PLCO study. Br J Cancer. 2012;107(1):207-214.

10. Veitonmaki T, Tammela TL, Auvinen A, Murtola TJ. Use of aspirin, but not other nonsteroidal anti-inflammatory drugs is associated with decreased prostate cancer risk at the population level. Eur J Cancer. 2013;49(4):938-945.

11. Liu Y, Chen JQ, Xie L, et al. Effect of aspirin and other non-steroidal anti-inflammatory drugs on prostate cancer incidence and mortality: a systematic review and metaanalysis. BMC Med. 2014;12:55.

12. Rothwell PM, Wilson M, Price JF, Belch JF, Meade TW, Mehta Z. Effect of daily aspirin on risk of cancer metastasis: a study of incident cancers during randomised controlled trials. Lancet. 2012;379(9826):1591-1601.

13. Choe KS, Correa D, Jani AB, Liauw SL. The use of anticoagulants improves biochemical control of localized prostate cancer treated with radiotherapy. Cancer. 2010;116(7):1820-1826.

14. Jacobs CD, Chun SG, Yan J, et al. Aspirin improves outcome in high risk prostate cancer patients treated with radiation therapy. Cancer Biol Ther. 2014;15(6):699-706.

This article is protected by copyright. All rights reserved 
15. Choe KS, Cowan JE, Chan JM, Carroll PR, D'Amico AV, Liauw SL. Aspirin use and the risk of prostate cancer mortality in men treated with prostatectomy or radiotherapy. J Clin Oncol. 2012;30(28):3540-3544.

16. Rothwell PM, Price JF, Fowkes FG, et al. Short-term effects of daily aspirin on cancer incidence, mortality, and non-vascular death: analysis of the time course of risks and benefits in 51 randomised controlled trials. Lancet. 2012;379(9826):1602-1612.

17. Osborn VW, Chen SC, Weiner J, Schwartz D, Schreiber D. Impact of aspirin on clinical outcomes for African American men with prostate cancer undergoing radiation. Tumori. 2016;102(1):65-70.

18. Zaorsky NG, Buyyounouski MK, Li T, Horwitz EM. Aspirin and statin nonuse associated with early biochemical failure after prostate radiation therapy. Int J Radiat Oncol Biol Phys. 2012;84(1):e13-17.

19. Hamstra DA, Conlon AS, Daignault $S$, et al. Multi-institutional prospective evaluation of bowel quality of life after prostate external beam radiation therapy identifies patient and treatment factors associated with patient-reported outcomes: the PROSTQA experience. Int J Radiat Oncol Biol Phys. 2013;86(3):546-553.

20. Steinberg GR, Dandapani M, Hardie DG. AMPK: mediating the metabolic effects of salicylate-based drugs? Trends Endocrinol Metab. 2013;24(10):481-487.

21. Salastekar N, Desai T, Hauser T, et al. Salsalate improves glycaemia in overweight persons with diabetes risk factors of stable statin-treated cardiovascular disease: A 30month randomized placebo-controlled trial. Diabetes Obes Metab. 2017;19(10):14581462.

22. Hawley SA, Fullerton MD, Ross FA, et al. The ancient drug salicylate directly activates AMP-activated protein kinase. Science. 2013;336(6083):918-922.

23. Troncone M, Cargnelli SM, Villani LA, et al. Targeting metabolism and AMP-activated kinase with metformin to sensitize non-small cell lung cancer (NSCLC) to cytotoxic therapy: translational biology and rationale for current clinical trials. Oncotarget. 2017;8(34):57733-57754.

24. Sanli T, Steinberg GR, Singh G, Tsakiridis T. AMP-activated protein kinase (AMPK) beyond metabolism: A novel genomic stress sensor participating in the DNA damage response pathway. Cancer Biol Ther. 2013;15(2).

25. Steinberg GR, Kemp BE. AMPK in Health and Disease. Physiol Rev. 2009;89(3):10251078.

26. O'Brien AJ, Villani LA, Broadfield LA, et al. Salicylate activates AMPK and synergizes with metformin to reduce the survival of prostate and lung cancer cells ex vivo through inhibition of de novo lipogenesis. Biochem J. 2015;469(2):177-187.

27. van Veelen $W$, Korsse SE, van de Laar L, Peppelenbosch MP. The long and winding road to rational treatment of cancer associated with LKB1/AMPK/TSC/mTORC1 signaling. Oncogene. 2011;30(20):2289-2303.

28. Fullerton MD, Galic S, Marcinko K, et al. Single phosphorylation sites in Acc1 and Acc2 regulate lipid homeostasis and the insulin-sensitizing effects of metformin. Nat Med. 2013.

This article is protected by copyright. All rights reserved 
29. Jones RG, Plas DR, Kubek $S$, et al. AMP-activated protein kinase induces a p53dependent metabolic checkpoint. Mol Cell. 2005;18(3):283-293.

30. Sanli T, Rashid A, Liu C, et al. Ionizing radiation activates AMP-activated kinase (AMPK): a target for radiosensitization of human cancer cells. Int J Radiat Oncol Biol Phys. 2010;78(1):221-229.

31. Storozhuk Y, Hopmans SN, Sanli T, et al. Metformin inhibits growth and enhances radiation response of non-small cell lung cancer (NSCLC) through ATM and AMPK. Br J Cancer. 2013;108(10):2021-2032.

32. Chou TC. Drug combination studies and their synergy quantification using the ChouTalalay method. Cancer Res. 2010;70(2):440-446.

33. Chou TC, Talalay P. Generalized equations for the analysis of inhibitions of MichaelisMenten and higher-order kinetic systems with two or more mutually exclusive and nonexclusive inhibitors. Eur J Biochem. 1981;115(1):207-216.

34. Smith BK, Ford RJ, Desjardins EM, et al. Salsalate (Salicylate) Uncouples Mitochondria, Improves Glucose Homeostasis, and Reduces Liver Lipids Independent of AMPK-beta1. Diabetes. 2016;65(11):3352-3361.

35. He $\mathrm{Y}$, Huang $\mathrm{H}$, Farischon $\mathrm{C}$, et al. Combined effects of atorvastatin and aspirin on growth and apoptosis in human prostate cancer cells. Oncol Rep. 2017;37(2):953-960.

36. Shi C, Zhang N, Feng Y, Cao J, Chen X, Liu B. Aspirin Inhibits IKK-beta-mediated Prostate Cancer Cell Invasion by Targeting Matrix Metalloproteinase-9 and Urokinase-Type Plasminogen Activator. Cell Physiol Biochem. 2017;41(4):1313-1324.

37. Fernandez HR, Linden SK. The aspirin metabolite salicylate inhibits lysine acetyltransferases and MUC1 induced epithelial to mesenchymal transition. Sci Rep. 2017;7(1):5626.

38. Kim KY, Seol JY, Jeon GA, Nam MJ. The combined treatment of aspirin and radiation induces apoptosis by the regulation of bcl-2 and caspase-3 in human cervical cancer cell. Cancer Lett. 2003;189(2):157-166.

39. Catton CN, Lukka H, Gu CS, et al. Randomized Trial of a Hypofractionated Radiation Regimen for the Treatment of Localized Prostate Cancer. J Clin Oncol. 2017;35(17):18841890.

40. Fleischman A, Shoelson SE, Bernier R, Goldfine AB. Salsalate improves glycemia and inflammatory parameters in obese young adults. Diabetes Care. 2008;31(2):289-294.

41. Goldfine $A B$, Fonseca $V$, Jablonski $K A$, et al. The effects of salsalate on glycemic control in patients with type 2 diabetes: a randomized trial. Ann Intern Med. 2010;152(6):346357.

42. Goldfine $A B$, Silver $R$, Aldhahi $W$, et al. Use of salsalate to target inflammation in the treatment of insulin resistance and type 2 diabetes. Clin Trans/ Sci. 2008;1(1):36-43.

43. Gwinn DM, Shackelford DB, Egan DF, et al. AMPK phosphorylation of raptor mediates a metabolic checkpoint. Mol Cell. 2008;30(2):214-226.

44. Taylor WR, Stark GR. Regulation of the G2/M transition by p53. Oncogene. 2001;20(15):1803-1815.

This article is protected by copyright. All rights reserved 
45. Laplante M, Sabatini DM. mTOR signaling in growth control and disease. Cell. 2012;149(2):274-293.

46. Sabatini DM. Twenty-five years of mTOR: Uncovering the link from nutrients to growth. Proc Natl Acad Sci U S A. 2017;114(45):11818-11825.

47. Laplante M, Sabatini DM. mTOR signaling at a glance. J Cell Sci. 2009;122(Pt 20):35893594. 


\section{Figure Legends}

Figure 1: Dose-Response effects of salicylate, RT and combined treatments on prostate cancer cell proliferation and clonogenic survival. A, B) Proliferation of PC3 cells seeded at 500 cells/well, expressed as fraction of control. Cells were pre-treated with SAL for 24-hours prior to treatment with indicated doses of RT. Results of 3 independent experiments ( 6 replicates each) are shown. The same set of data is plotted as a function of RT (A) or SAL (B) dose. C) Combination index analysis was conducted with the proliferation data shown in A and B, using the Chou-Talalay method and the CompuSyn software. Combination indexes (CI) are plotted against effect size (Fa: the calculated level of inhibition) for the range of RT dose (indicated in legend) and SAL doses (written above/below respective data point). CI>1 represents antagonistic, $\mathrm{CI}=1$ additive, $\mathrm{CI}<1$ synergistic relationships. D-E) Clonogenic survival of PC3 (D) and LNCaP (E) cells. Cells (PC3: 500/well) or (LNCaP:1500/well) were seeded in 12-well plates, allowed to adhere overnight and treated with indicated SAL doses for 24-hours prior to RT. Cells grew for 7 (PC3) or 14 (LNCaP) days, and stained with crystal violet stain. Colonies greater than 50 cells were counted as viable. F) Effects of low-dose SAL (50 $\mu \mathrm{M})$ on a range of RT doses, was examined with clonogenic assays. Increasing seeding density of cells were used for increasing doses of RT; PC3 (500-8000/well) and LNCaP (1500-8000/well) were seeded in 12-well plates, allowed to adhere overnight and treated with indicated SAL doses for 24-hours prior to RT. Colony counts were normalized to number of cells seeded per well, and then to untreated control conditions. Statistical analysis of proliferation and clonogenic data was carried out with Prism6, 2-way ANOVA with Fisher-LSD to test for significance at $\mathrm{p}<0.05(*)$. 
Figure 2: Effects of salsalate on in vivo prostate cancer tumor growth. PC3 xenograft response to $2.5 \mathrm{~g} / \mathrm{kg}$ salsalate (dimerized SAL pro-drug) in chow diet and a RT (single fraction of 10Gy). Balb/c-nude mice (n=6/group) were injected subcutaneously in the flank with $1 \times 10^{6}$ PC3 cells suspended in $100 \mu \mathrm{L}$ of warm PBS, and allowed to engraft and grow. A) Study timeline and interventions. When tumors reached an average volume of $100 \mathrm{~mm}^{3}(5 \mathrm{wks})$, measured using a hand caliper and the ellipsoid volume calculation $\mathrm{V}=0.5\left(\mathrm{~L} * \mathrm{~W}^{2}\right)$, animals were randomized into control (chow) or salsalate diet (Chow $+2.5 \mathrm{~g} / \mathrm{kg}$ Salsalate). After 1 week on diet, mice were further randomized into Control (sham RT) or RT (10 Gy) treatment. RT was delivered with conformal parallel opposed pair (POP) fields using established dosimetric methods (30). B,C) Tumor volume was measured over time in non-RT mice (B) and RT mice (C). D) Exact ex-vivo endpoint tumor volumes were measured after tumors were excised. E) FFPE cross-sections of PC3 xenografts were stained with hematoxylin and eosin stain. Statistical analysis was carried out with Prism6, 2-way ANOVA with Fisher-LSD to test for significance at $\mathrm{p}<0.05$ (*).

\section{Figure 3: Modulation of the ATM-AMPK/ACC-mTOR pathway by SAL and RT.}

A-E) Acute (1-hour, in blue) and chronic (48-hour, in red) signaling responses to RT were assessed in PC3 cells. PC3 cells were seeded in 6-well plates, allowed to adhere for 24h, followed by RT treatment ( 2 Gy or $8 \mathrm{~Gy}$ ). Cells were allowed to grow for the indicated times ( 1 or $48 \mathrm{~h}$ ), lysed, snap frozen, and prepared for electrophoresis and immunoblotting for markers in the ATM, AMPK and mTOR pathways. F-I) Acute 1-hour response of PC3 cells to 8 Gy RT after 24-hour SAL pre-treatment (in red) compared to 0 Gy control (in blue) was assessed by immunoblotting. J-L) Chronic, 48-hour response of PC3 cells to SAL with 0 Gy control (in blue) 
or 2 Gy (in red) RT was assessed by immunoblotting to examine modulation of AMPK, mTOR, and Erk signaling. M) De novo lipogenesis was evaluated $48 \mathrm{~h}$ after treatments (matching immunoblotting in J-L), using radiolabelled ${ }^{3} \mathrm{H}$-acetate uptake in PC3 cells treated with SAL and RT (2 Gy), as indicated above. N) Proposed model of signaling and metabolic events activated by SAL and RT in PrCa cells. All densitometry of immunoblotting experiments were normalized to untreated controls, analyzed using Image $J$ software, and represent $n=3-5$ independent experiments. 2-way ANOVA was used to test for significance with Fisher LSD testing, and significance was considered at $\mathrm{p}<0.05 .^{*} \mathrm{p}<0.05, * * \mathrm{p}<0.01, * * * \mathrm{p}<0.001, * * * * \mathrm{p}<0.0001$.

Figure s1. Clonogenic survival of PNT1A cells. Normal human prostate epithelial PNT1A cells we subjected to clonogenic survival assays, as indicated in Materials and Methods, while treated with the indicated concentrations of salicylate (SAL). Normalized average + SE values of 3 independent experiments are shown.

Figure s2. Assessment of proportion of surviving tumor tissue with high and low cellular density and necrosis or fibrosis, in tumors assessed at end point. Formalin Fixed Paraffin Embedded tumors were cross-sectioned, mounted on to glass slides and stained with H+E. Three to four cross-sectional area slides were obtained from each tumor treated with SAL, RT, both or neither, scanned and quantified with Image $\mathbf{J}$ software for areas of live tumor, necrosis and fibrosis, as indicated in A. Results from calculated areas were normalized against the total tumor area in each slide (B and C).

This article is protected by copyright. All rights reserved 
Figure s3. Effects of salicylate (SAL) and radiotherapy (RT) on Erk, ATM and p53 phosphorylation or total levels in PC3 and LNCaP prostate cancer cells. Cells were preincubated with the indicated concentrations of SAL for $24 \mathrm{~h}$ before treatment with indicated doses of RT. Subsequently, cells were incubated for either $1 \mathrm{~h}$ (B) or $48 \mathrm{~h}$ (A) before lysis. Immunoblotting analysis was pursued with the indicated antibodies, as described in Methods. Representative results of 2-3 experiments are shown. 

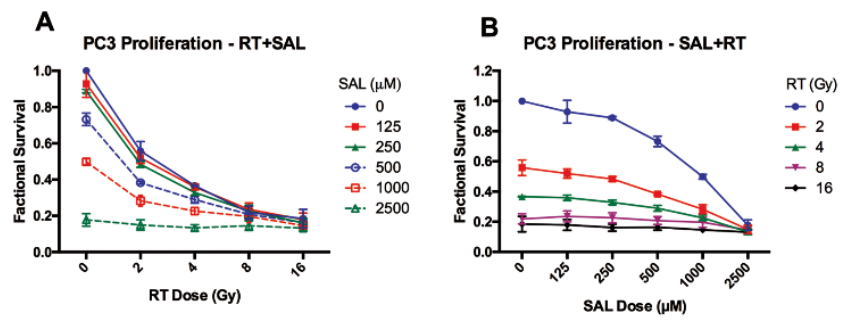

C Combination Index and Effect Size
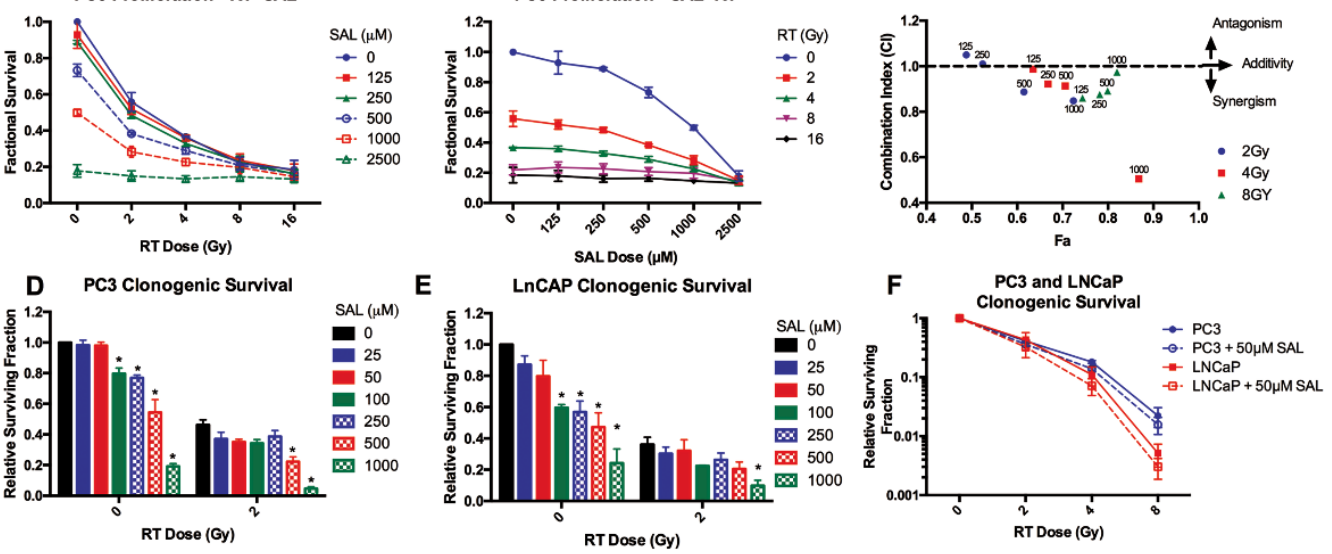

Figure 1

This article is protected by copyright. All rights reserved 


\section{A}
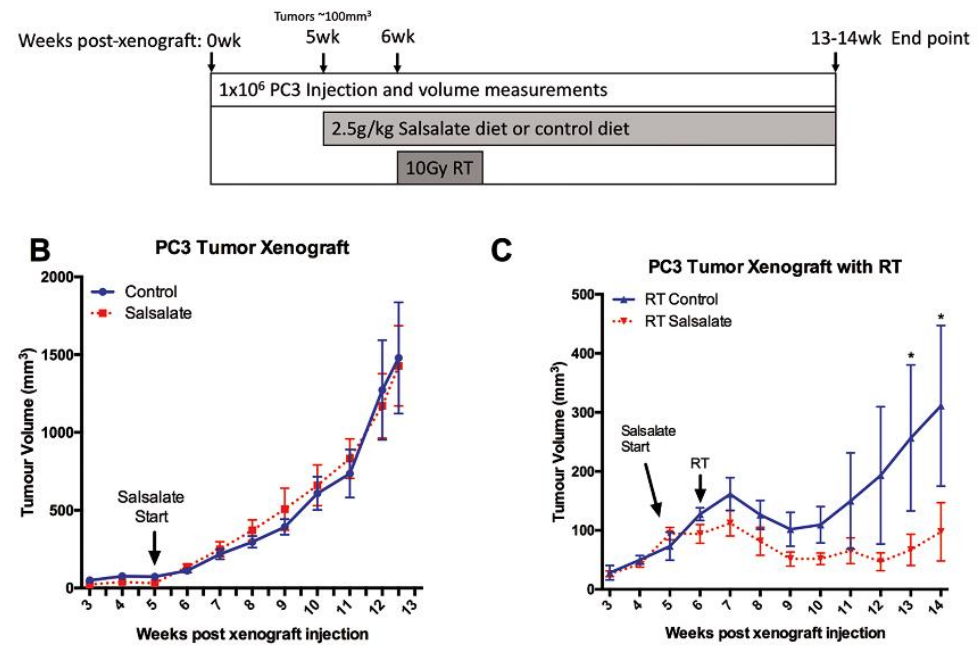

C

PC3 Tumor Xenograft with RT

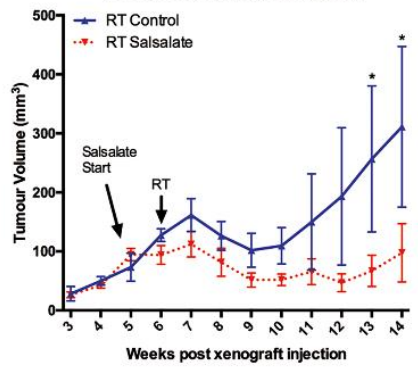

D

E
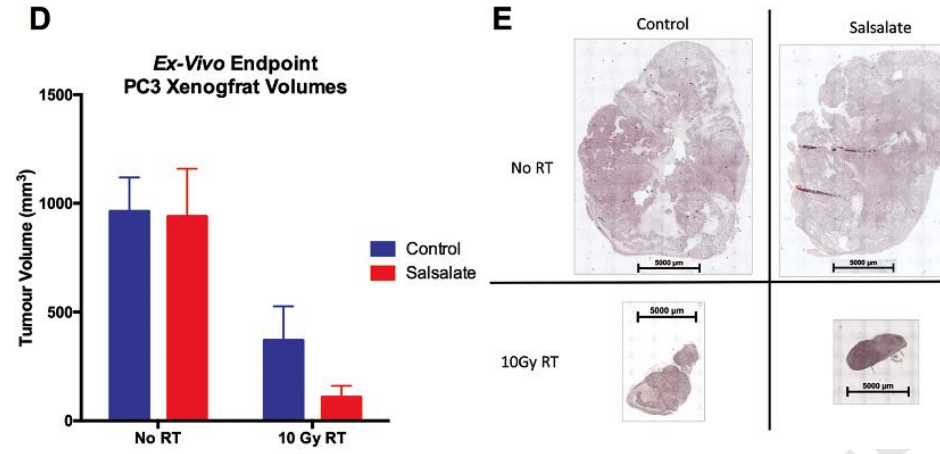

No RT

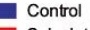
- Salsalate
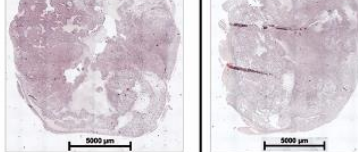

10Gy RT

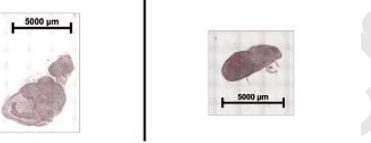

Figure 2 


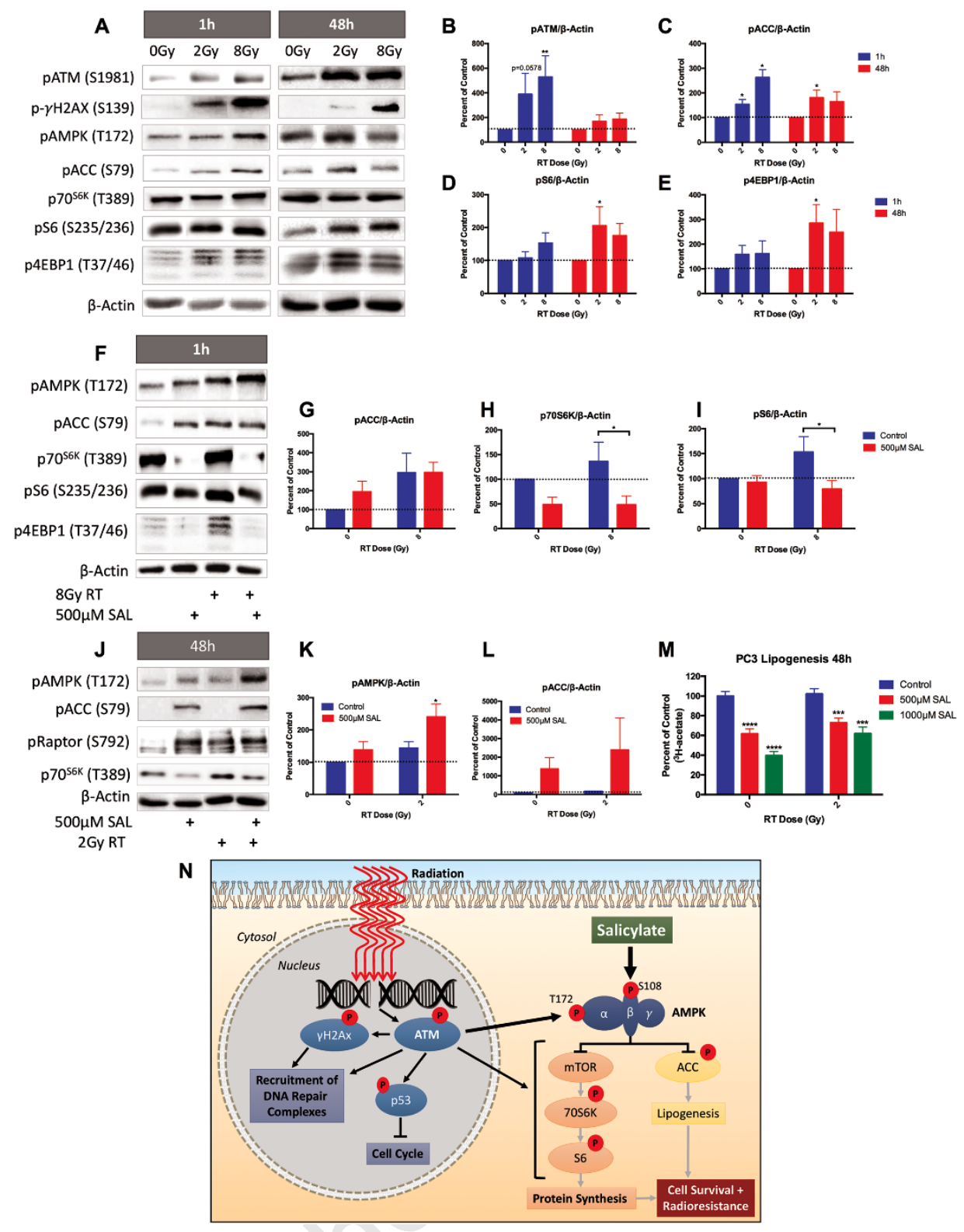

Figure 3

This article is protected by copyright. All rights reserved 


\section{University Library}

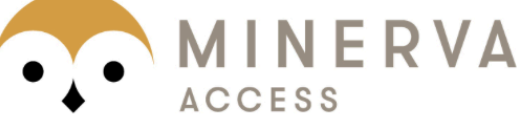

A gateway to Melbourne's research publications

Minerva Access is the Institutional Repository of The University of Melbourne

Author/s:

Broadfield, LA;Marcinko, K;Tsakiridis, E;Zacharidis, PG;Villani, L;Lally, JSV;Menjolian,

G;Maharaj, D;Mathurin, T;Smoke, M;Farrell, T;Muti, P;Steinberg, GR;Tsakiridis, T

Title:

Salicylate enhances the response of prostate cancer to radiotherapy.

Date:

2019-04

\section{Citation:}

Broadfield, L. A., Marcinko, K., Tsakiridis, E., Zacharidis, P. G., Villani, L., Lally, J. S. V., Menjolian, G., Maharaj, D., Mathurin, T., Smoke, M., Farrell, T., Muti, P., Steinberg, G. R. \& Tsakiridis, T. (2019). Salicylate enhances the response of prostate cancer to radiotherapy.. Prostate, 79 (5), pp.489-497. https://doi.org/10.1002/pros.23755.

Persistent Link:

http://hdl.handle.net/11343/285247 